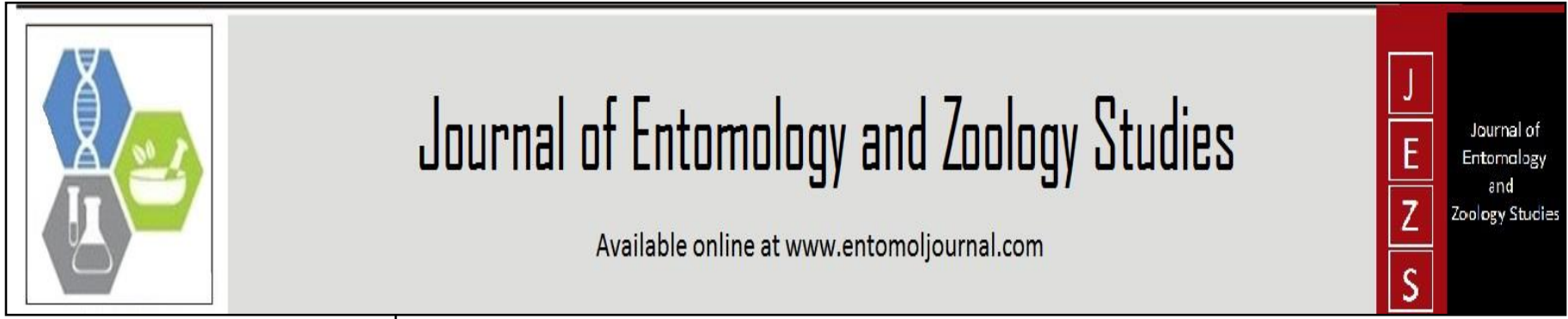

E-ISSN: 2320-7078

P-ISSN: 2349-6800

www.entomoljournal.com JEZS 2021; 9(4): 106-110 (C) 2021 JEZS

Received: 07-05-2021

Accepted: 09-06-2021

Anthea Uloma Ikpo

Department of Parasitology and

Entomology, Nnamdi Azikiwe

University, Awka, Nigeria

Obioma Chebechi Nwaorgu

Department of Parasitology and

Entomology, Nnamdi Azikiwe

University, Awka, Nigeria

Kindness Chidi Irikannu

Department of Parasitology and

Entomology, Nnamdi Azikiwe

University, Awka, Nigeria

Joseph Uche Anumba

Federal Ministry of Health,

National Arbovirus and Vectors

Research Centre, Enugu, Nigeria

Lilian Chiamaka Ezemuoka

Department of Parasitology and

Entomology, Nnamdi Azikiwe

University, Awka, Nigeria

Chikodili Obiajulu Aniefuna Department of Parasitology and Entomology, Nnamdi Azikiwe

University, Awka, Nigeria
Corresponding Author: Kindness Chidi Irikannu Department of Parasitology and Entomology, Nnamdi Azikiwe University, Awka, Nigeria

\section{Molecular characterization of Anopheles gambiae s.l. from three senatorial districts in Enugu State, Southeastern Nigeria}

\author{
Anthea Uloma Ikpo, Obioma Chebechi Nwaorgu, Kindness Chidi \\ Irikannu, Joseph Uche Anumba, Lilian Chiamaka Ezemuoka and \\ Chikodili Obiajulu Aniefuna
}

DOI: https://doi.org/10.22271/j.ento.2021.v9.i4b.8787

\begin{abstract}
A study of molecular characterization of Anopheles gambiae sensu lato, from three senatorial districts in Enugu State, was conducted between May, 2019 and June, 2020. The aim was to determine the siblings' species of the mosquito complex and the distribution of the S (Savannah) and M (Mopti) molecular forms within the An. gambiae sensu stricto in the area. Three randomly selected communities; Awkunanaw (Enugu East), Obukpa (Enugu North) and Awgu (Enugu West senatorial districts) were used as sampling sites. Anopheles gambiae s.l. larvae were collected by dip method and reared to adult for the study. A total of 450(100\%) An. gambiae s.l. mosquitoes were subjected to Polymerase Chain Reaction (PCR). Of this number, 150(33.33\%) were randomly selected from bulk of An. gambiae s.l. mosquitoes collected from each of the three communities. All the 450(100\%) were identified as An. gambiae s.s. The Restriction Fragment Length Polymorphism (RFLP) was done on the 450(100\%) An. gambiae s.s. A total of 300(66.67\%) from Awkunanaw and Obukpa Nsukka communities were An. gambiae S-form while 150(33.33\%) from Awgu were identified as An. gambiae M-form. The study has revealed that An. gambiae s.s. is the dominant malaria vector within the complex in the area. Integrated vector control is recommended.
\end{abstract}

Keywords: mosquitoes, molecular, PCR, Anopheles gambiae, Enugu

\section{Introduction}

Anopheles gambiae s.l. is a vector of the malaria disease in Africa [1]. Vector control is the major way to prevent and reduce malaria transmission. Two major approaches to vector control includes the use of Insecticide-Treated Nets (ITNs) and Indoor Residual Spraying (IRS). Sleeping under an ITN can reduce contact between mosquitoes and humans by providing both a physical barrier and an insecticidal effect. Population-wide protection can result from the killing of mosquitoes on a large scale where there is high access and usage of such nets within a community ${ }^{[2]}$. Indoor residual spraying involves spraying the inside of housing structures with an insecticide, typically once or twice per year ${ }^{[2]}$.

The proper identification of Anopheles mosquito species is important in the control of the malaria disease. Some mosquito vectors are species complexes that are difficult to separate morphologically. For example, the An. gambiae s.l. species complex comprises of at least seven sibling species that are morphologically indistinguishable namely; An. gambiae s.s., An. arabiensis, An. quadriannulatus species A, An. quadriannulatus species $\mathrm{B},{ }^{[3-5]}$, An. melas, An. merus and An. bwambae. Three additional species were later described: An. comorensis, An. amharicus, and An. coluzzii ${ }^{[6]}$. The aim of this study was to determine the An. gambiae s.l. siblings species present in three senatorial zones in Enugu State and the distribution of the S (Savannah) and M (Mopti) molecular forms within the An. gambiae s.s.

\section{Materials and Methods}

\section{Study Area}

Enugu State is in the southeastern Nigeria and lies between the coordinates of latitude $6^{0} 27^{\prime} 35.87^{\prime \prime} \mathrm{N}$ and longitude $7^{0} 32$ '56.22'"E. 
The state has total area of 7,618 sq. Km. Politically the state is divided into three senatorial districts. The inhabitants of state are predominantly Igbos with a population of at least 4,881,500 people ${ }^{[7]}$. They are mainly farmers, traders and civil servants. The climate of the area is tropical and vegetation characteristics are mainly rain forest with an average annual rainfall of about $1300 \mathrm{~mm}$ and average daily atmospheric temperature of $30{ }^{0} \mathrm{C}$. There are two distinct seasons, the wet (March - October) and dry season (November - February). The study was conducted in 3 randomly selected communities in the senatorial districts.

\section{Community Visitation and Sensitization}

A letter of introduction obtained from the Department of Parasitology and Entomology, Nnamdi Azikiwe University was used to make advocacy visits to the leaders of the study communities. They were properly sensitized about the mode and intent of the study. Their permission was also obtained to use their environment for the study.

\section{Study Design}

Randomized controlled trail study design was employed with communities as units of study. Three communities (one per senatorial district), were randomly selected. This was done by writing names of all the communities in each of the senatorial districts on sheets of paper. The papers were placed in a bag and one community was blindly picked to represent a senatorial district. The randomly selected communities and their geographical coordinates were; Awkunanaw (Enugu East senatorial district) 6.3907N, 7.4760E; Obukpa (Enugu North senatorial district) 6.890N, 7.4003E; and Awgu (Enugu West senatorial district) 6.0640N, 7.4760E. Anopheles mosquito larvae sampling was conducted in the communities between May, 2019 and June, 2020.

\section{Larval Sampling}

Larval collection was done using $350 \mathrm{ml}$ standard dipper ${ }^{[8]}$. Ladles and pipettes were also used. Collections were done in all forms of breeding sites including puddles, rice fields and other farms, containers, excavations, tire tracks, hoof prints and crab holes amongst others. When Anopheles mosquito larvae are present, 10-30 dips were taken depending on the size of larval habitat. Where dipping was not possible, for example, when larvae were seen on polythene, can or leaf, the larvae were transferred from the breeding material into the collection container. Sampling was carried out twice daily, in the morning between 7:00am to 11:00am and in the evening between $4: 00 \mathrm{pm}$ to $6: 00 \mathrm{pm}$ (local time) for about 30 minutes in each larval habitat for 5 days. All larvae and pupae collected were transported in containers labeled according to the site of collection to the insectary of the National Arbovirus and Vectors Research Centre, Enugu, Nigeria, were they were reared to adults, for molecular studies. The larvae were fed on fish feed (Tropical Flakes, Samyu Pets Corp and Shulin City) in bowls measuring $500 \mathrm{ml}$, while the adult mosquitoes were fed with a $10 \%$ sugar solution in cotton wool on emergence. Relative humidity and temperature of $80 \pm$ $10 \%$ and $25 \pm 2{ }^{\circ} \mathrm{C}$, respectively were maintained in the insectary throughout the period.

\section{Morphological Identification of Anopheles gambiae s.l. mosquito species}

Adult An. gambiae s.l. species used in the study were identified at the Entomology Laboratory of the National
Arbovirus and Vectors Research Centre, Enugu using morphological keys ${ }^{[9-11]}$.

\section{Molecular Identification of Anopheles gambiae s.l. mosquito species}

The molecular study was conducted at the Molecular Laboratory of the National Arbovirus and Vector Research Center, Enugu. Polymerase chain reaction (PCR) was used to identify sibling species in the An. gambiae s.l. complex. The identified An. gambiae s.s. were further subjected to restriction fragment length polymorphic polymerase chain reaction RFLP-PCR to separate of $\mathrm{M}$ and $\mathrm{S}$ molecular forms.

\section{Deoxyribonucleic Acid Extraction}

Deoxyribonucleic Acid (DNA) from the mosquitoes were extracted using Quick-DNA ${ }^{\mathrm{TM}}$ Tissue/Insect Miniprep Kit manual Catalog No. D6016 produced by Zymo Research, USA 2019.

\section{Procedure for Deoxyribonucleic Acid Extraction}

Five mosquitoes from same senatorial districts were placed in a $1.5 \mathrm{ml}$ Eppendorf tube and 50ul BashingBead ${ }^{\mathrm{TM}}$ Buffer added to the Eppendorf tube and grounded slowly and properly with a grinding stick. Three hundred and fifty (350ul) of BashingBead ${ }^{\mathrm{TM}}$ Buffer was added to the tube to bring the volume to $400 \mathrm{ul}$ and the tube was vortex to permit total mixing of the solution. ZR BashingBead ${ }^{\mathrm{TM}}$ Lysis Tube $(2.0 \mathrm{~mm})$ was centrifuged in a microcentrifuge at $10,000 \mathrm{rpm}$ for 1 minute. The supernatant was transferred to a ZymoSpin $^{\mathrm{TM}}$ III-F Filter placed in a Collection Tube and then centrifuged at 8,000 rpm for 1 minute. The collection tube was collected with the filtrate and Zymo-Spin ${ }^{\mathrm{TM}}$ III-F Filter was discarded. A measure of 1,200ul of Genomic Lysis Buffer was added to the filtrate in the Collection Tube and the mixture vortex for 10 seconds. The Zymo-Spin ${ }^{\mathrm{TM}}$ IICR Column 1 was placed in a Collection Tube and 800ul (the remaining mixture was not discarded as it was used at a later stage) of the mixture from the previous step was transferred to it and the mixture was centrifuged at 10,000 rpm for I minute. The flow was discarded through filtrate from the Collection Tube and the Zymo-Spin ${ }^{\mathrm{TM}}$ IICR Column 1 placed in the Collection Tube. The remaining mixture from the previous stage was transferred to Zymo-Spin ${ }^{\mathrm{TM}}$ Column 1 and centrifuge at 10,000 rmp for 1 minute. The flow was discarded from the Collection Tube through the filtrate and the ZymoSpin $^{\mathrm{TM}}$ IICR Column 1 placed in a new Collection Tube. Two hundred (200ul) DNA Pre-Wash Buffer was added to the Zymo-Spin ${ }^{\mathrm{TM}}$ IICR Column in the new Collection Tube and centrifuged at 10,000rmp for 1 minute. A measure of 500ul gDNA Wash Buffer was added to the Zymo-Spin ${ }^{\mathrm{TM}}$ IICR Columnin the Collection Tube and centrifuged at 10,000 rpm for 1 minute. The Zymo-Spin ${ }^{\mathrm{TM}}$ IICR Column was transferred to a clean $1.5 \mathrm{ml}$ Eppendorf tube and 100ul DNA Elution Buffer added to it. The Zymo-Spin ${ }^{\mathrm{TM}}$ IICR in the Eppendorf tube was centrifuged at $10,000 \mathrm{rpm}$ for 30 seconds to elute the DNA. The eluted solution in the Eppendorf tube containing the mosquito DNA was stored at $-20^{\circ} \mathrm{C}$ for subsequent PCR analysis (Quick-DNA ${ }^{\mathrm{TM}}$ Tissue/Insect Miniprep Kit Manual Catalog No. D6016).

\section{Anopheles gambiae s.l. sibling species Identification by} Polymerase Chain Reaction

Deoxyribonucleic acid extract from the above described procedure was used for the identification of mosquito 
siblings' species. Mosquito DNA was amplified by PCR using Quik-load one Taq Master mix with standard buffer by New England Biolab (NEB), USA. PCR tubes were labeled according to the three senatorial districts and laced on ice pack. Reagents were added in the following order (Master mixing reaction set up includes); One Taq $2 x$ Master mix (14ul), UN (universal forward primer) 2ul, GA (reverse primer for An. gambiae s.s.) 2ul, ME (reverse primer for $A n$. melas) 2ul, AR (reverse primer for An. arabiensis) 2ul, QD (reverse primer for An quadriannulatus) 2ul, Nuclease free water $21 \mathrm{ul}$ and DNA template $5 \mathrm{ul}$. Total reaction volume was 50ul. The tubes were inserted into the thermal cycler and ran under the following conditions (35 cycles); predenaturation $95{ }^{\circ} \mathrm{C}$ for 5 minutes, Denaturation $94{ }^{\circ} \mathrm{C}$ for 30 seconds, Annealing $68{ }^{\circ} \mathrm{C}$ for 30 seconds, Elongation $72{ }^{\circ} \mathrm{C}$ for 60 seconds and Final elongation $72{ }^{\circ} \mathrm{C}$ for 5 mimutes [12, 13]. Primer sequence used are; UN (universal forward primer) 5'GTG TGC TTC CTC GAT GT-3', GA (reverse primer for An. gambiae s.s.) 5'CTG GTT TGG TCG GCA CGT TT-3', ME (reverse primer for An. melas) 5'TGA CCA ACC CAC TCC CTT GA-3', AR (reverse primer for An. arabiensis) AAG TGT CCT TCT CCA TCC TA -3', QD (reverse primer for An. quadriannulatus) 5'CAG ACC AAG ATG GTT AGT AT -3 ,

\section{Gel Electrophoresis}

Two percent $(2 \%)$ agarose gel was prepared and used to run electrophoresis for a period of 1 hour 30 minutes at 120 volts. Gel was stained red by gel red (ethidium bromide) for 10 minutes. The band was visualized with Dark reader transilluminator. The expected band size for An. gambiae s.s. is 390bp, An. arabiensis 315bp, An. melas 464 bp, and An. quadriannulatus $153 \mathrm{bp}$.

\section{Restriction Fragment Length Polymorphism RFLP on Anopheles gambiae s.s.}

Restriction fragment length polymorphism (RFLP) was done to digest the fragment revealing different forms ( $M$ and $\mathrm{S}$ forms) of An. gambiae s.s. at different size pattern. The RFLP was processed by placing 16ul of PCR product into a clean $1.5 \mathrm{ml}$ micro-centrifuge tube and adding $2 \mathrm{ul}$ of $10 \mathrm{x}$ restriction buffer to the same tube. A measure 1ul of Hahl (20 units) restriction enzyme was added to the same tube and the mixture was thoroughly mixed and centrifuge for 10 seconds to obtain the mixture at the bottom of the tube. This was incubated at $37{ }^{\circ} \mathrm{C}$ for 3 hours. The digest product was run on $2 \%$ agarose gel at 120 volts for 1 hour 30 minutes to verify different size patterns ${ }^{[12]}$.

\section{Data Analysis}

Descriptive statistics was used to analyze the data obtained from the study.

\section{Results}

A total of 450(100\%) An. gambiae s.l. complex mosquitoes were subjected to PCR for molecular identification. Of this number, 150(33.33\%) were randomly selected from the bulk of An. gambiae s.l. mosquitoes collected from each of the study community. After PCR, all the 450(100\%) were molecularly identified as An. gambiae s.s. No other siblings species of the An. gambiae s.l. complex was identified (Table 1).

Table 1: Sibling Species of Anopheles gambiae s.l identified by PCR across the Zones

\begin{tabular}{|c|c|c|c|c|}
\hline Mosquito spp. & Obukpa Nsukka & Awgu & Awkunanaw & Total \\
\hline An. gambiae s.s. & 150 & 150 & 150 & $450(100 \%)$ \\
\hline An. melas & 0 & 0 & 0 & $0(0.00)$ \\
\hline An. quadrinnulatus & 0 & 0 & 0 & $0(0.00)$ \\
\hline An. arabiensis & 0 & 0 & 0 & $0(0.00)$ \\
\hline TOTAL & $150(33.33 \%)$ & $150(33.33 \%)$ & $150(33.33 \%)$ & $450(100 \%)$ \\
\hline
\end{tabular}

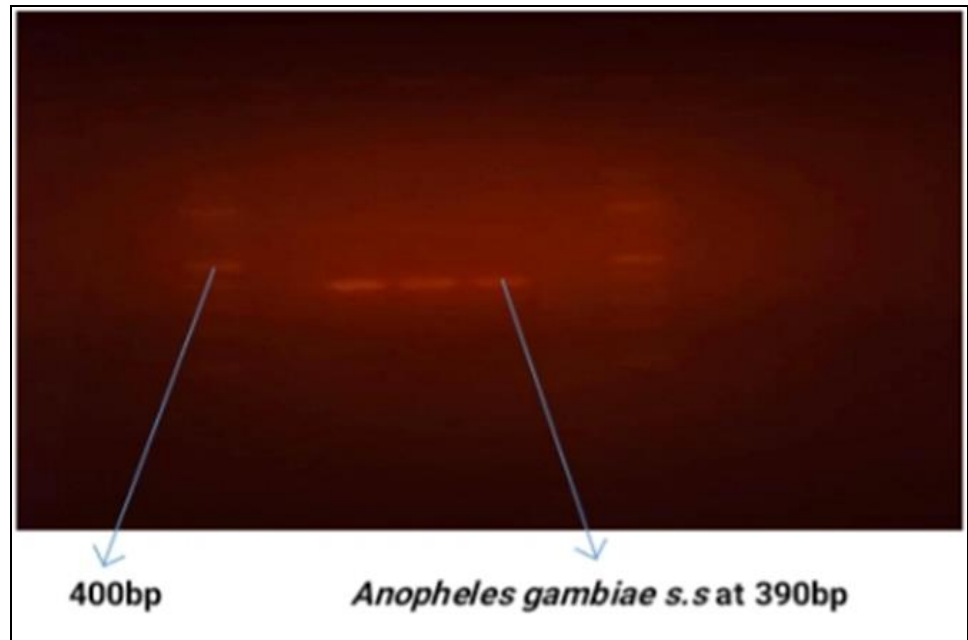

Plate 1: Electrophoresis gel image of Anopheles gambiae s.l. speciation

The Restriction Fragment Length Polymorphism (RFLP) was done on the $450(100 \%)$ An. gambiae s.s. A total of $300(66.67 \%)$ from Awkunanaw and Obukpa Nsukka communities were An. gambiae S form while 150(33.33\%) An. gambiae s.s. from Awgu were identified as An. gambiae M (Table 2). 
Table 2: Distribution of the molecular forms ( $\mathrm{S}$ and $\mathrm{M}$ forms) of An. gambiae s.s.

\begin{tabular}{|c|c|c|c|c|}
\hline & Obukpa Nsukka & Awgu & Awkunanaw & Total (\%) \\
\hline An. gambiae S form (\%) & 150 & 0 & 150 & $300(66.67 \%)$ \\
\hline An. gambiae M form (\%) & 0 & 150 & 0 & $150(33.33)$ \\
\hline Total (\%) & $150(33.33)$ & $150(33.33)$ & $150(33.33)$ & $450(100 \%)$ \\
\hline
\end{tabular}

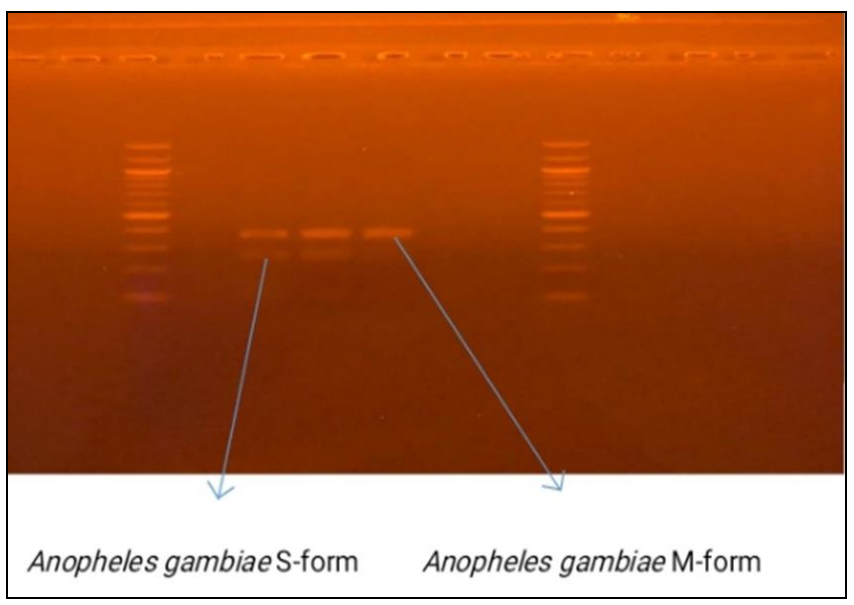

Plate 2: Electrophoresis Result of the Molecular Forms of Anopheles gambiae s.s.

\section{Discussion}

Polymerase Chain Reaction speciation of the An. gambiae s.l. species complex identified only An. gambiae s.s. as the only siblings' species of the complex present in the three senatorial districts in the study area. This compares favourably with another study in the country ${ }^{[14]}$, where two siblings species; An. gambiae s.s. and An. coluzzii were reported, with An. gambiae s.s. being the dominant species. Also, the presence of An. gambiae s.s. in Imo, Anambra and Abia States has been reported ${ }^{[3,14]}$ while An. coluzzii was only reported in Ebonyi State ${ }^{[14]}$. This study reported allopatric breeding for the An. gambiae s.s. in the study area which agrees with another study in southeastern Nigeria ${ }^{[14]}$. In Ibadan Nigeria, An. gambiae s.s. and An. coluzzii were also reported with An. gambiae s.s. also being the dominant species, however, sympathric breeding of the two sibling species was reported [15].

Further studies on An. gambiae s.s. using the RFLP-PCR revealed the presence of both $\mathrm{M}$ and $\mathrm{S}$ molecular forms in the study area. The $\mathrm{M}$ forms were detected in Awgu in while the $\mathrm{S}$ forms were observed in Awkunanaw and Obukpa, Nsukka. This is in tandem with another study in Nigeria ${ }^{[16]}$ where the distribution of the molecular forms also varied, with the $S$ form being the dominant species mostly reported in southern region of the country (mangrove, forest and other transitional ecological zones). The $\mathrm{M}$ forms were mainly reported in the northern region (guinea and Sudan savanna ecological zones) ${ }^{[16]}$. In another study ${ }^{[17]}, \mathrm{S}$ molecular form of An. gambiae s.s. also had a wider distribution across Nigeria compared to the latter. Both molecular forms occur throughout the country with no apparent relationship to the ecological transition from dry savannah in the north to humid forest in the southern Nigeria ${ }^{[17]}$. There is no sympatric association in this study as both molecular form breed in allopatry as was also reported in another study ${ }^{[14]}$. The $\mathrm{S}$ form of the An. gambiae s.s. has been associated with malaria distribution and the $\mathrm{M}$ form with lymphatic filariasis distribution but the part played by these two molecular forms in malaria and/or lymphatic filariasis is still largely unknown ${ }^{[16]}$.

\section{Conclusion}

The study has revealed that An. gambiae s.s. is the only or the dominant species within the An. gambiae s.l. complex in the study area. This suggest that malaria transmission in the area may largely be by An. gambiae s.s. Integrated vector control which may include special interventions with IRS and subsidized ITNs is recommended.

\section{References}

1. Egbuche CM, Onyido AE, Umeanaeto PU, Nwankwo EN, Omah IF, Ukonze CB et al. Anopheles species composition and some climatic factors that influence their survival and population abundance in Anambra East LGA, Anambra State, Nigeria. Nigerian Journal of Parasitology 2020;42(2):240-250.

2. WHO. World Malaria Report. Geneva: World Health Organization; 2018, 32-59. https ://www.who.int/malar ia/publications/world -malaria-report-2018/en/. Accessed 11 Mar 2020.

3. Irikannu $\mathrm{KC}$, Onyido AE, Umeanaeto PU, Onwube O, Ogaraku JC, Egbuche CM et al. Molecular characterization and malaria transmission potential of Anopheles gambiae complex in Awka, Anambra state, Nigeria. International Journal of Mosquito Research. 2019;6(6):96-101.

4. Coetzee M. Distribution of the African malaria vectors of the Anopheles gambiae complex. American Journal of Tropical Medicine and Hygiene 2004;70:103-104.

5. Coetzee M, Craig M, le Sueur D. Distribution of African malaria mosquitoes belonging to the Anopheles gambiae complex. Parasitology Today 2000;16:74-77.

6. Yakob L. Epidemiological consequences of a newly discovered cryptic subgroup of Anopheles gambiae Biol Lett 2011;7(6):947-9.

7. NPC-National Population Commission. Population Census of Nigeria. Population distribution in Local Government Areas by Sex and Number of Households. 2006.

8. WHO. World Health Organisation recommended insecticides for indoor residual spraying against malaria vectors 2013. Available online at: http://www.who.int/whopes/Insecticides_IRS_Malaria_2 5_Oct_2013.pdf Accessed on 28/3/2018.

9. Gillies MT, de Meillon B. The Anophelinae of Africa South of the Sahara. Publ South African Inst Med Res. 1968;54:1-343.

10. Gillet JD. Common African Mosquitoes and their Medical Importance. William Heinemann Medical Books Limited, London. 1972, 36

11. Gillies MT, Coetzee BA. Supplementary to Anophelinae of Africa, South of Sahara (Afro- Tropical Region), South Africa Institute of Medical Research 1987;55:1143.

12. Fanello C, Santolamazza F, Della A. Simultaneous Identification of Species and Molecular forms of Anopheles gambiae complex by PCR-RFLP Med Vet Entomol 2002;16:461-464.

13. Higa Y, Tomas T, Tsuda Y, Miyagi I. A multiplex PCR- 
based molecular identification of five morphologically related, medically important subgenus Siegomyia mosquitoes from the Genus Aedes (Diptera: Cullicidae) found in the Ryukyu Archipelago, Japan Jananesse Journal of Infectious Disease 2010;64:312-316.

14. Chukwuekezie O, Nwosu E, Nwangwu O, Dogunro F, Onwude C, Agashi N et al. Resistance status of Anopheles gambiae (s.l.) to four commonly used insecticides for malaria vector control in South-East Nigeria. Parasites \& Vectors 2020;13:152.

15. Okorie PN, Ademowo OG, Irving H, Kelly-Hope LA, Wondji CS. Insecticide susceptibility of Anopheles coluzzii and Anopheles gambiae mosquitoes in Ibadan, Southwest Nigeria. Med Vet Entomol. 2015;29:44-50.

16. Okorie PN, McKenzie FE, Ademowo GO, Bockarie M, Kelly-Hope L. Nigeria Anopheles Vector Database: An overview of 100 years' Reasearch PLOS ONE: 2011;6(12):e28347 dol: 1371/journal.pone 0028347

17. Onyabe DY, Vajime CA, Nock IH, Ndams SI, Akpa AU, Aliriba AA et al. The distribution of $\mathrm{M}$ and $\mathrm{S}$ molecular forms of Anopheles gambiae in Nigeria. Trans R Soc Trop Med Hyg 2003;97:605-608. 